\title{
Self-force on a scalar charge in radial infall from rest using the Hadamard-WKB expansion
}

\author{
Paul R. Anderson* \\ Department of Physics, Wake Forest University, Winston-Salem, NC 27109, USA \\ Ardeshir Eftekharzadeh $\dagger$ and B. L. Hu \\ Department of Physics, University of Maryland, College Park, MD 20742, USA
}

(Dated: September 29, 2005)

\begin{abstract}
We present an analytic method based on the Hadamard-WKB expansion to calculate the self-force for a particle with scalar charge that undergoes radial infall in a Schwarzschild spacetime after being held at rest until a time $t=0$. Our result is valid in the case of short duration from the start. It is possible to use the HadamardWKB expansion in this case because the value of the integral of the retarded Green's function over the particle's entire past trajectory can be expressed in terms of two integrals over the time period that the particle has been falling. This analytic result is expected to be useful as a check for numerical prescriptions including those involving mode sum regularization and for any other analytical approximations to self-force calculations.

PACS numbers: 04.25.Nx, 04.30.Db, 04.70.Bw
\end{abstract}

\section{INTRODUCTION}

This latest version has a new appendix C containing the Erratum in Ref. [41] to the version published in Physical Review D [40]. In this version we have made the corrections indicated therein.

Computation of the self-force for a point particle in orbit around a black hole is a topic of

*Electronic address: anderson@wfu.edu

†Electronic address: eftekhar@wam.umd.edu

${ }^{\ddagger}$ Electronic address: hub@physics.umd.edu 
active research today [1, 2, 3] prompted by the preparation of gravitational wave detectors such as LISA which are capable of detecting gravitational waves emitted when a compact object falls into a supermassive black hole [4, 5$]$.

An exact expression for the self-force in a black hole spacetime has been obtained only for the cases of a scalar or electric charge held at rest in a Schwarzschild spacetime [6, 7, 8, 9] and an electric charge held at rest on the symmetry axis of a Kerr spacetime [10, 11]. Approximate analytical expressions have been obtained using Green's functions for scalar, electric, and gravitational charges in various weak field limits [12, 13, 14]. Most other calculations have involved the use of mode sum techniques [15, 16] in cases of high symmetry such as a static charge [17, 18, 19], radial infall [20, 21], a circular orbit [22, 23, 24, 25], or a slightly elliptical orbit [24].

Since the mode-sum regularization procedure has been developed extensively, aiming at practical calculations, it is important to find independent ways to check its accuracy and reliability. This of course also applies to any other method, numerical or analytical that might be developed in the future. To date the checks on various mode-sum regularization procedures which we are aware of fall into three categories: 1) comparisons with exact analytical results, 2) comparisons with analytical approximations, 3) comparisons of the results of one mode-sum regularization technique with those of another. Most of the comparisons that have been made fall into the third category [21, 23, 24, 25, 26, 27]. Comparisons with exact analytical results are of course the most reliable but also the most limited. They have only been done in the case of static scalar and electric charges held at rest in a Schwarzschild spacetime [17]. Comparison with an analytic approximation in the weak field limit has been made for a static scalar charge at rest in an axisymmetric spacetime [19]. In this paper we present results which can be used as a check in the second category. However, unlike most previous analytical approximations to the self-force, ours is valid in the strong field as well as the weak field limit. Specifically, we consider the case of a particle with scalar charge which is held at rest until a time $t=0$ and subsequently falls radially towards a Schwarzschild black hole.

In a previous paper [28] (which we will refer to as Paper I) we introduced an approximation for the computation of the self-force of a scalar charge in a Schwarzschild spacetime using the Hadamard-WKB expansion [29, 30, 31, 32, 33, 34, 35] which can account for the radiation reaction effects from the charge's recent past history. This is an expansion for the tail 
term part of the retarded Green's function and is valid in a spacetime region close to the charge. A similar approach has been taken to compute part of the gravitational self-force in an arbitrary spacetime by Anderson, Flanagan,and Ottewill [36]. In Paper I an eighth order WKB expansion was used to compute the nonvanishing part of the tail term to order $\left(x-x^{\prime}\right)^{6}$. Since then we have obtained results to order $\left(x-x^{\prime}\right)^{14}$ using a 16th order WKB expansion.

Recently Anderson and Wiseman [37] investigated the convergence of the HadamardWKB expansion for the self-force. They posited that convergence is obtained as long as the point separation in proper time is small enough so that the null geodesics emanating from the point at the earlier time have not had time to re-intersect the particle's path. We know the absence of caustics is perhaps the least stringent criterion for the validity of a quasilocal expansion (e.g., the domain of validity of Riemann normal coordinates). After computing this proper time separation for the case of a particle orbiting a black hole in a circular path, they use the result of Paper I [Hadamard-WKB expansion to order $\left(x-x^{\prime}\right)^{6}$ ] to evaluate the convergence of the series expansion for the self-force.

Anderson and Wiseman go on to show that the primary constituent of the self-force comes from the tail term of the Green's function when the points are too widely separated for this expansion to converge. This would seem to rule out the possibility of using the Hadamard-WKB expansion by itself to compute the self-force, which is not surprising, as the quasilocal expansion has this intrinsic limitation to begin with.

However, we want to point out that there is at least one case in which it is possible to determine the entire self-force using the Hadamard-WKB expansion. If the particle is held at rest until a time $t=0$ after which it falls radially towards the black hole, then the entire self-force can be determined using the Hadamard-WKB expansion so long as the particle has not fallen too far from its starting point. While the range over which the particle falls must be small, the results can serve as a useful check on other methods of computing the self-force.

In Section [1 a brief review is given of the general method we use to compute the self-force. In Section [III the self-force is computed for the case in which a particle is held at rest until time $t=0$ and subsequently falls towards the event horizon of a Schwarzschild black hole. We derive a closed expression for the self-force in terms of integrals over the tail part of the retarded Green's function when the points are split by only a small amount. The splitting 
is between the point where the particle is at a time $t>0$ and various other points inside the past lightcone of this point. We then use the Hadamard-WKB expansion described in Paper I to derive power series expressions for the nonzero components of the self-force. In Section IV the series are evaluated for a specific example. The results are given in Table I and Figure I. Some details of the calculations are given in Appendices A and B and the coefficients derived for the series are also given in Appendix B.

\section{REVIEW OF METHOD}

Consider a massless scalar field with a source term consisting of a point particle of scalar charge $q$. The wave equation is $[2,8]^{1}$

$$
\begin{aligned}
\square \Phi(x) & =-\rho(x) \\
\rho(x) & =q \int_{-\infty}^{\infty} \frac{\delta^{4}(x, z(\tau))}{\sqrt{-g}} d \tau
\end{aligned}
$$

where $\tau$ is the proper time and $z(\tau)$ is the trajectory of the particle. A formal solution to this equation can be obtained using the retarded Green's function:

$$
\Phi(x)=q \int_{-\infty}^{\infty} G_{R}\left(x, z\left(\tau^{\prime}\right)\right) d \tau^{\prime} .
$$

The self-force is given formally by

$$
f_{\mu}(\tau)=q\left[\nabla_{\mu} \Phi(x)\right]_{x=z(\tau)} .
$$

This expression is divergent and must be regularized. Quinn [2] has shown that the regularized expression for the self-force can be split into a local term plus a finite integral over the gradient of the retarded Green's function. The latter is often called the "tail term".

The Hadamard expansion for the retarded Green's function is [2, 32, 33, 35] $]^{2}$

$$
G_{R}\left(x, x^{\prime}\right)=\theta\left(x, x^{\prime}\right)\left\{\frac{u\left(x, x^{\prime}\right)}{4 \pi} \delta\left[\sigma\left(x, x^{\prime}\right)\right]-\frac{v\left(x, x^{\prime}\right)}{8 \pi} \theta\left[-\sigma\left(x, x^{\prime}\right)\right]\right\} .
$$

\footnotetext{
${ }^{1}$ Note that our conventions differ from those of [2, 8]. One can obtain those of Ref. 2] by letting $\Phi \rightarrow$ $\Phi / \sqrt{4 \pi}, q \rightarrow \sqrt{4 \pi} q$, and $G_{R} \rightarrow G_{R} /(4 \pi)$. Those of Ref. [8] can be obtained by letting $\Phi \rightarrow-\Phi / \sqrt{4 \pi}$ and $q \rightarrow \sqrt{4 \pi} q$.

${ }^{2}$ In Eq. (1.1) of Paper I this equation was written with an incorrect factor of $\sigma\left(x, x^{\prime}\right)$ in the denominator of the first term. The relationships between our definitions of $u$ and $v$ and those of Ref. [2] were also given incorrectly. Taking into account the previously mentioned difference in the definition of the retarded Green's function, the correct relationship is that the definition of $u$ in Eq. (4) is equivalent to that in Ref. [2] while the definition of $v$ differs from that of Ref. [2] by a factor of $1 / 2$. No other equations in Paper I are affected and the results remain unchanged.
} 
Here $\theta\left[-\sigma\left(x, x^{\prime}\right)\right]$ is defined to be one if the point $x^{\prime}$ is inside the light cone of the point $x$ and zero otherwise, while $\theta\left(x, x^{\prime}\right)$ is defined to be one if the point $x$ resides in the future of a spacelike hypersurface involving the point $x^{\prime}$ and zero otherwise. The quantity $\sigma\left(x, x^{\prime}\right)$ is equal to one-half the square of the proper distance between $x$ and $x^{\prime}$ along the shortest geodesic connecting them. The function $v\left(x, x^{\prime}\right)$ contributes to the tail part of the self-force [2]. It obeys the equation

$$
\square_{x} v\left(x, x^{\prime}\right)=0
$$

and is symmetric under the exchange of the two points, i.e. $v\left(x, x^{\prime}\right)=v\left(x^{\prime}, x\right)$. In a general spacetime [30, 31]

$$
v(x, x)=-\frac{1}{6} R(x) .
$$

The tail term for the self-force obtained from Eqs. (11) and (21) can be written in the form (see, e.g., [37])

$$
\begin{aligned}
\left(f_{\mu}(\tau)\right)_{\text {tail }}= & -\frac{q^{2}}{8 \pi} \int_{\tau_{0}}^{\tau}\left(\frac{\partial}{\partial x^{\mu}} v\left[x, z\left(\tau^{\prime}\right)\right]\right)_{x=z(\tau)} d \tau^{\prime} \\
& +q^{2} \int_{-\infty}^{\tau_{0}}\left(\frac{\partial}{\partial x^{\mu}} G_{R}\left[x, z\left(\tau^{\prime}\right)\right]\right)_{x=z(\tau)} d \tau^{\prime} .
\end{aligned}
$$

It is necessary that $\tau_{0}$ be chosen so that the Hadamard-WKB expansion for $v$ is valid throughout the region of integration of the first integral in Eq. (7).

An expansion for $v\left(x, x^{\prime}\right)$ in Schwarzschild spacetime was found in Paper I using a WKB expansion for the Euclidean Green's function. The WKB expansion is obtained via an iteration procedure and is increased by two orders upon each iteration. From a WKB expansion of order $(2 N)$ one can obtain an expansion for $v\left(x, x^{\prime}\right)$ that includes terms up to order $\left(x-x^{\prime}\right)^{2 N-2}$. For the metric

$$
d s^{2}=-(1-2 M / r) d t^{2}+d r^{2} /(1-2 M / r)+r^{2} d \Omega^{2},
$$

the expansion for $v$ is of the form

$$
v\left(x, x^{\prime}\right)=\sum_{i, j, k=0}^{\infty} v_{i j k}(r)\left(t-t^{\prime}\right)^{2 i}(\cos \gamma-1)^{j}\left(r-r^{\prime}\right)^{k}
$$

with

$$
\cos \gamma \equiv \cos \theta \cos \theta^{\prime}+\sin \theta \sin \theta^{\prime} \cos \left(\phi-\phi^{\prime}\right) .
$$

We have recently found that it is possible to extend this expansion to order $\left(x-x^{\prime}\right)^{2 N-1}$ by using the fact that $v\left(x, x^{\prime}\right)$ is a symmetric function. If one takes $(2 N-1)$ partial derivatives 
of $v\left(x, x^{\prime}\right)$ with respect to some combination of the coordinates $x^{a}$ and sets $x^{\prime}=x$ then by symmetry it must be true that taking the same combination of derivatives with respect to $x^{\prime a}$ and setting $x^{\prime}=x$ results in an equivalent expression. Using the expansion (9) results in the coefficients of terms of odd powers of $\left(x-x^{\prime}\right)$ being expressed in terms of the coefficients of terms of smaller even powers. The coefficients of terms of even powers of $\left(x-x^{\prime}\right)$ cannot be obtained in this way and must be obtained from the WKB expansion.

\section{COMPUTATION OF THE SELF-FORCE}

We now proceed to compute the self-force for a particle of mass $m$ and scalar charge $q$ falling radially from rest. We assume that the particle has been held fixed at $r=r_{0}, \theta=\theta_{0}$, and $\phi=\phi_{0}$ from $t=-\infty$ to $t=0$ which corresponds to proper time $\tau=0$. At time $t=0$ the particle is released, with no initial velocity, and subsequently falls radially towards a Schwarzschild black hole of mass $M$. We shall denote the spacetime point at which it is released by $y_{0}$. Our objective is to calculate the self-force exerted on the particle at a proper time $\tau>0$ when the particle is at the point $y=\left(t, r, \theta_{0}, \phi_{0}\right)$.

The first term in Eq. (7) can be computed using the Hadamard-WKB expansion. The results of this computation are discussed below. The second term is more difficult. We next show that it too can be computed using the Hadamard-WKB expansion due to the fact that the charge is stationary until it begins falling at time $t=0$.

First consider the problem of computing the field $\Phi_{\text {static }}$ at the point $y$ due to a completely static charge $q$ at the position $r=r_{0}, \theta=\theta_{0}, \phi=\phi_{0}$. The solution to this problem in a Schwarzschild spacetime has been given by Wiseman [8]. For the above locations of the charge and field point, with a metric of the form (8), and taking into account a difference in conventions, it can be written as

$$
\Phi_{\text {static }}(y)=\frac{1}{4 \pi} q \sqrt{1-\frac{2 M}{r_{0}}} \frac{1}{r_{0}-r} .
$$

On the other hand one can use Eq. (2), to write the field due to the static charge as

$$
\Phi_{\text {static }}(y)=q \int_{-\infty}^{\infty} G_{R}\left[t, r ; t^{\prime}\left(\tau^{\prime}\right), r_{0}\right] d \tau^{\prime} .
$$

Here and for the rest of this section we suppress the dependence of various quantities on the angles $\theta_{0}$ and $\phi_{0}$. Dividing the integration region in the same way as was done in Eq. (7) 
and using the Hadamard expansion (4) one can write this latter equation as

$$
\begin{aligned}
\Phi_{\text {static }}(y) & =\frac{q}{4 \pi} \sqrt{1-\frac{2 M}{r_{0}}} \int_{0}^{t} u\left[t, r ; t^{\prime}, r_{0}\right] \delta\left[\sigma\left(t, r ; t^{\prime}, r_{0}\right)\right] d t^{\prime} \\
& -\frac{q}{8 \pi} \sqrt{1-\frac{2 M}{r_{0}}} \int_{0}^{t_{R}} v\left[t, r ; t^{\prime}, r_{0}\right] d t^{\prime}+q \int_{-\infty}^{0} G_{R}\left[t, r ; t^{\prime}\left(\tau^{\prime}\right), r_{0}\right] d \tau^{\prime}
\end{aligned}
$$

In the first two terms the integration variable has been changed from $\tau^{\prime}$ to $t^{\prime}$. The two theta functions in Eq. (4) result in an upper limit for the second integral which is equal to the retarded time $t_{R}$ which is given in Eq. (A12). The time $t$ is taken to be the time that it would take a particle to fall from $r_{0}$ to $r$ assuming that it starts at rest. Then the third term on the right in Eq. (13) is the same, except for the gradient and a factor of $q$, as the second term on the right in Eq. (77). The value of this term can be obtained by computing the other three terms in the equation. The term on the left is given in Eq. (11) and the second term on the right can be computed using the Hadamard-WKB expansion.

To calculate the first term on the right in Eq. (13), we note that the argument of the delta function vanishes on the light cone of the point $y$. Since the charge is static

$$
\begin{aligned}
\sigma\left(t, r ; t^{\prime}, r_{0}\right) & =\sigma\left(t, r ; t_{R}, r_{0}\right)+\left.\left(\frac{\partial}{\partial t^{\prime}} \sigma\left(t, r ; t^{\prime}, r_{0}\right)\right)\right|_{t^{\prime}=t_{R}}\left(t^{\prime}-t_{R}\right)+\ldots \\
& =\sigma_{t_{R}}\left(t^{\prime}-t_{R}\right)+\ldots
\end{aligned}
$$

where the shorthand notation $\sigma_{\mu} \equiv \sigma_{; \mu}$ has been used. Then

$$
\delta\left(\sigma\left(t, r ; t_{R}, r_{0}\right)\right)=\delta\left[\sigma_{t_{R}}\left(t^{\prime}-t_{R}\right)\right]=\frac{\delta\left(t^{\prime}-t_{R}\right)}{\left|\sigma_{t_{R}}\right|} .
$$

Next one must calculate $u\left(y, y_{R}\right)$ with $y$ and $y_{R} \equiv\left(t_{R}, r_{0}, \theta_{0}, \phi_{0}\right)$ connected by a null radial geodesic. By substituting the Hadamard expansion into the equation satisfied by the Green's function it is possible to show that in general [29, 30, 31]

$$
\begin{aligned}
u\left(x, x^{\prime}\right) & =\Delta^{1 / 2}\left(x, x^{\prime}\right) \\
\Delta\left(x, x^{\prime}\right) & =-\frac{\operatorname{det}\left(-\sigma_{; \mu \nu^{\prime}}\right)}{\sqrt{-g(x)} \sqrt{-g\left(x^{\prime}\right)}}
\end{aligned}
$$

Thus what remains is to calculate $\sigma_{t_{R}}$ and $\sigma_{; \mu \nu^{\prime}}$ for the two points $x=y$ and $x^{\prime}=y_{R}$. Although there may be some simple way to reason out the answer, as shown in Appendix $\mathrm{A}$, it can be obtained by solving the geodesic equations and integrating the result to obtain the proper distance along the geodesic. The result is

$$
\begin{aligned}
\sigma_{t_{R}}\left(t, r ; t_{R}, r_{0}\right) & =r_{0}-r \\
u\left(t, r ; t_{R}, r_{0}\right) & =1 .
\end{aligned}
$$


Substituting Eq. (17) into Eq. (13) and computing the integral one finds that

$$
\int_{-\infty}^{0} G_{R}\left[t, r ; t^{\prime}\left(\tau^{\prime}\right), r_{0}\right] d \tau^{\prime}=\frac{1}{8 \pi} \sqrt{1-\frac{2 M}{r_{0}}} \int_{0}^{t_{R}} v\left[t, r ; t^{\prime}, r_{0}\right] d t^{\prime}
$$

With the definitions

$$
\begin{aligned}
& \Phi_{s}(y)=\frac{q}{8 \pi} \sqrt{1-\frac{2 M}{r_{0}}} \int_{0}^{t_{R}} v\left[t, r ; t^{\prime}, r_{0}\right] d t^{\prime} \\
& \Phi_{f}(y)=\frac{q}{8 \pi} \int_{0}^{\tau} v\left[t, r ; t^{\prime}\left(\tau^{\prime}\right), r^{\prime}\left(\tau^{\prime}\right)\right] d \tau^{\prime}
\end{aligned}
$$

Eq. (17) becomes

$$
f_{\mu}(\tau)=q\left[\frac{\partial}{\partial y^{\mu}}\left(\Phi_{s}(y)-\Phi_{f}(y)\right)\right]
$$

Here the facts that in a Schwarzschild spacetime $v(x, x)=0$ and (as shown by our expansion) $v\left(y, y_{R}\right)=0$, have been used to interchange the order of integration and differentiation. Note that this derivation only works for the time and radial components of the self-force. Because of spherical symmetry, the angular components of the self-force for a radial trajectory are zero. Finally the subscript "tail" has been dropped because for a geodesic trajectory the local part of the self-force is zero in a Schwarzschild spacetime [2].

As a result of Eq. (20), the problem of calculating the self-force reduces to calculating $\Phi_{s}$ and $\Phi_{f}$. This is an exact result. We now calculate the right hand side of Eq. (20) using the Hadamard-WKB expansion for $v\left(x, x^{\prime}\right)$ whose form is given in Eq. (91). For radial geodesics, $\cos \gamma=1$, so only the coefficients $v_{i 0 k}(r)$ contribute. The result for $\Phi_{s}$ is

$$
\Phi_{s}(y)=\frac{q}{8 \pi} \sqrt{1-\frac{2 M}{r_{0}}} \sum_{i, k=0}^{\infty}\left(\frac{1}{2 i+1}\right) v_{i 0 k}(r)\left[t^{2 i+1}-\left(t-t_{R}\right)^{2 i+1}\right]\left(r-r_{0}\right)^{k}
$$

To calculate $\Phi_{f}$ one can use the geodesic equations (A7) to convert the integral (19b) to an integral over the radial coordinate $r$. One can further solve the geodesic equations to obtain the trajectory $t(r)$. After substituting the Hadamard-WKB expansion for $v$, the integral (19b) can be computed numerically.

An alternative is to expand all relevant quantities in both $\Phi_{s}$ and $\Phi_{f}$ in Taylor series about $r_{0}$. This allows one to compute the integrals analytically order by order. The derivation is 
given in more detail in Appendix B. We find

$$
\begin{aligned}
f_{t}(\tau) & =\frac{q^{2}}{22400 \pi r_{0}^{2}} \sqrt{1-\frac{2 M}{r_{0}}}\left(40-\frac{106 M}{r_{0}}\right)\left(\frac{r_{0}-r}{r_{0}}\right)^{3}+O\left[\left(\frac{r_{0}-r}{r_{0}}\right)^{4}\right] \\
f_{r *}(\tau) & =-\frac{3 q^{2}}{11200 \pi r_{0}^{2}} \sqrt{\frac{2 M}{r_{0}}}\left(1-\frac{2 M}{r_{0}}\right)\left(\frac{r_{0}-r}{r_{0}}\right)^{5 / 2}+O\left[\left(\frac{r_{0}-r}{r_{0}}\right)^{7 / 2}\right]
\end{aligned}
$$

with $r *$ the Regge-Wheeler coordinate defined by

$$
r^{*} \equiv r+2 M \log \left(\frac{r-2 M}{2 M}\right) .
$$

It turns out that each subsequent order of the WKB expansion adds another term to the series. Using a 16th order WKB expansion we have results for a total of six terms in the expansions for both $f_{t}$ and $f_{r *}$. The coefficients of these terms are displayed in Appendix B.

\section{SPECIFIC EXAMPLE}

As a specific example we have chosen the case in which a particle begins falling from rest at $r *=40 M$ at time $t=\tau=0$, with $r *$ defined in Eq. (23). Using the series in Appendix B, the $f_{t}$ and $f_{r} *$ components of the self-force have been computed. The results are displayed in Table I and Figure I. The relative errors in the table are estimated by taking the absolute value of the ratio between the last term used in the series expansion (C1) for a given component of the self-force and the entire series for that component.

The expected convergence distance, obtained by measuring how far the particle will fall in the time for a null geodesic leaving the location of the particle at time $t=0$ to circle the black hole and intersect the particle in a new location, is $r \approx 30.7 \mathrm{M}$. On the other hand, an examination of the error shows that more terms than the six we have computed for each component of the self-force are necessary for an accurate determination of the self-force when $r<33 M$.

While the range over which we can compute the self-force is relatively small and the calculation is for a particle held at rest that subsequently undergoes radial infall in a Schwarzschild spacetime, the merit of this method is it produces reliable results and thus can provide an independent check on other (existing and future) prescriptions for the calculation of the self-force. It is worth noting that our calculation started with a finite result and therefore no regularization was needed. The generalization to the cases where the self-force is due to electromagnetism and gravity should be straight-forward. 


\begin{tabular}{|c|c|c|c|c|}
\hline$r / M$ & $10^{14}\left(M^{2} / q^{2}\right) f_{t}$ & Error & $10^{14}\left(M^{2} / q^{2}\right) f_{r^{*}}$ & Error \\
\hline 34.25 & 6.93066 & $6.9 \times 10^{-6}$ & -5.7384 & $3.4 \times 10^{-5}$ \\
\hline 34.00 & 120.72 & $4.4 \times 10^{-4}$ & -96.78 & $1.4 \times 10^{-3}$ \\
\hline 33.75 & 616 & $3.4 \times 10^{-3}$ & -520 & $8.4 \times 10^{-3}$ \\
\hline 33.50 & 2080 & 0.013 & -1840 & 0.25 \\
\hline 33.25 & 5680 & 0.031 & -5200 & 0.052 \\
\hline 33.00 & 13800 & 0.059 & -13200 & 0.089 \\
\hline 32.75 & 30000 & 0.10 & -30000 & 0.13 \\
\hline 32.50 & 66000 & 0.14 & -62000 & 0.18 \\
\hline
\end{tabular}

TABLE I: The dependence of the temporal $\left(f_{t}\right)$ and radial $\left(f_{r^{*}}\right)$ components of the self-force on the radial distance $r / M$ are given for a particle undergoing radial infall after being held at rest until a time $t=0$. The particle's initial location is at $r^{*}=40 M$ which corresponds to $r \approx 34.43 M$. The error shown for each case is an estimate of the relative error and is obtained by taking the absolute value of the ratio of the last term used in the series for the self-force to the entire series.

\section{Acknowledgments}

Some of this work was done while P. R. A. was visiting Los Alamos National Laboratory. He would like to thank E. Mottola and the entire T8 Group for their hospitality. This work was supported in part by the National Science Foundation under grant PHY03-00710.

\section{APPENDIX A: CALCULATION OF $u$ AND $\sigma_{t_{R}}$}

In this appendix we calculate the quantities $u\left(y, y_{R}\right)$ and $\sigma_{t_{R}}\left(y, y_{R}\right)$ with $y=\left(t, r, \theta_{0}, \phi_{0}\right)$ and $y_{R}=\left(t_{R}, r_{0}, \theta_{0}, \phi_{0}\right) ; t_{R}$ is the retarded time defined in Eq. (A12). The points $y$ and $y_{R}$ are separated by a radial null geodesic. From Eqs. (14) and (16) it is clear that

$$
\begin{aligned}
u\left(y, y_{R}\right) & =\left[\frac{-\operatorname{det}\left(-\sigma_{; \mu \nu^{\prime}}\left(x, x^{\prime}\right)\right)}{\sqrt{-g(x)} \sqrt{-g\left(x^{\prime}\right)}}\right]_{x \rightarrow y, x^{\prime} \rightarrow y_{R}}^{1 / 2} \\
\sigma_{t_{R}}\left(y, y_{R}\right) & =\left[\frac{\partial}{\partial t^{\prime}} \sigma\left(x, x^{\prime}\right)\right]_{x \rightarrow y, x^{\prime} \rightarrow y_{R}} .
\end{aligned}
$$

Although the points are separated by a radial null geodesic, it is necessary to assume a more general separation before computing derivatives. Once this is done then the specific 


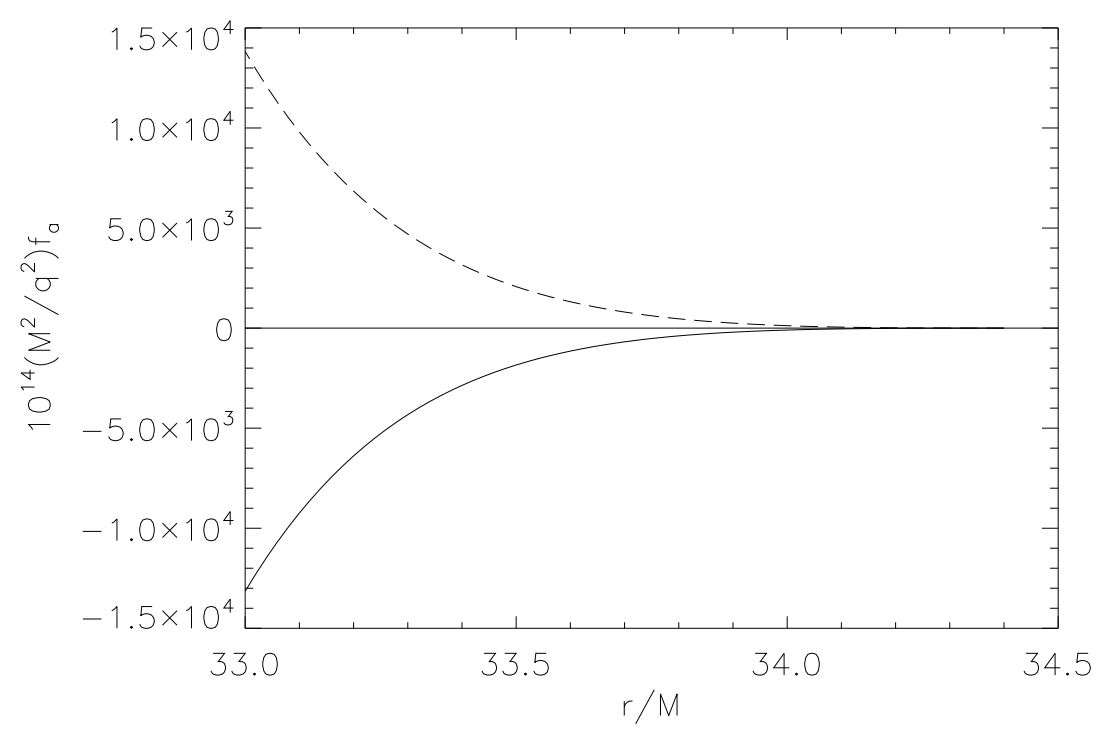

FIG. 1: In this figure the two components of the self-force are plotted for the case of a particle undergoing radial infall after being held at rest until a time $t=0$. The particle's initial location is at $r^{*}=40 M$ which corresponds to $r \approx 34.43 M$. The dashed curve corresponds to $f_{t}$ and the solid curve to $f_{r^{*}}$.

separation can be taken. Because of spherical symmetry the angular dependence of the quantity $\sigma\left(x, x^{\prime}\right)$ must be such that it is a function of $\cos \gamma$ which is defined in Eq. (10). To see this assume that the two points have an angular separation of $\gamma$. Since any arbitrary rotation of the coordinate system does not change this angle, the angular dependence of $\sigma$ can only be through $\gamma$. Because any $2 \pi$ rotation around the origin of the coordinate system should render $\sigma$ unchanged, $\sigma$ must be a periodic function of $\gamma$ with period $2 \pi$. This dependence can be written as a Fourier series in $\sin n \gamma$ and $\cos n \gamma$. The coefficients of the sine terms must identically vanish since $\sigma\left(x, x^{\prime}\right)$ is a symmetric function in $x$ and $x^{\prime}$ and switching $x$ and $x^{\prime}$ changes the sign of $\gamma$. Since $\cos n \gamma$ can be written in terms of powers of $\cos \gamma$ [38], it is then clear that $\sigma=\sigma\left(t, r ; t^{\prime}, r^{\prime} ; \cos \gamma\right)$.

We next compute the angular derivatives of $\sigma$ beginning with

$$
\sigma_{; \theta}\left(x, x^{\prime}\right)=\left(\frac{\partial \sigma}{\partial \cos \gamma}\right)\left(-\sin \theta \cos \theta^{\prime}+\cos \theta \sin \theta^{\prime} \cos \left(\phi-\phi^{\prime}\right)\right) \text {. }
$$

Note that in general $\sigma_{; \mu}\left(x, x^{\prime}\right)$ is a vector at $x$ and a scalar at $x^{\prime}$. Next we can proceed to 
take a second derivative.

$$
\begin{aligned}
\sigma_{; \theta \theta^{\prime}}\left(x, x^{\prime}\right)= & \left(\frac{\partial^{2} \sigma}{\partial(\cos \gamma)^{2}}\right)\left(-\sin \theta \cos \theta^{\prime}+\cos \theta \sin \theta^{\prime} \cos \left(\phi-\phi^{\prime}\right)\right)^{2} \\
& +\left(\frac{\partial \sigma}{\partial \cos \gamma}\right)\left(\sin \theta \sin \theta^{\prime}+\cos \theta \cos \theta^{\prime} \cos \left(\phi-\phi^{\prime}\right)\right)
\end{aligned}
$$

For the special case of a radial geodesic, $\theta^{\prime}=\theta, \phi^{\prime}=\phi$, and thus $\cos \gamma=1$. As a result

$$
\left(\sigma_{; \theta \theta^{\prime}}\right)_{\cos \gamma=1}=\left(\frac{\partial \sigma}{\partial \cos \gamma}\right)_{\cos \gamma=1} .
$$

Similarly it is easy to show that

$$
\left(\sigma_{; \phi \phi^{\prime}}\right)_{\cos \gamma=1}=\sin ^{2} \theta\left(\frac{\partial \sigma}{\partial(\cos \gamma)}\right)_{\cos \gamma=1}
$$

and

$$
\begin{aligned}
& \left(\sigma_{; \theta t^{\prime}}\right)_{\cos \gamma=1}=\left(\sigma_{; \theta r^{\prime}}\right)_{\cos \gamma=1}=\left(\sigma_{; \theta \phi^{\prime}}\right)_{\cos \gamma=1}=0 \\
& \left(\sigma_{; \phi t^{\prime}}\right)_{\cos \gamma=1}=\left(\sigma_{; \phi r^{\prime}}\right)_{\cos \gamma=1}=\left(\sigma_{; \phi \theta^{\prime}}\right)_{\cos \gamma=1}=0
\end{aligned}
$$

Along with the other combinations of radial and time derivatives it is still necessary to calculate $\left(\frac{\partial \sigma}{\partial(\cos \gamma)}\right)_{\cos \gamma=1}$. If we choose coordinates such that $\theta=\theta^{\prime}=\pi / 2$ then from Eq. (A4b)

$$
\left(\frac{\partial \sigma}{\partial(\cos \gamma)}\right)_{\cos \gamma=1}=\left(\sigma_{; \phi \phi^{\prime}}\right)_{\phi^{\prime}=\phi} .
$$

To calculate the rest of the derivatives that comprise $\sigma_{; \mu \nu^{\prime}}$ it is useful to write $\sigma$ in terms of the proper time $\tau$ and to assume that the points both lie in the equatorial plane so that $\theta=\theta^{\prime}=\pi / 2$. Since $\sigma$ is one-half the square of the proper distance between the two points along the shortest geodesic connecting them we have

$$
\sigma=-\frac{1}{2} \tau^{2}
$$

The proper time $\tau$ can be computed by solving the geodesic equations [39]

$$
\begin{aligned}
\tau & =-\sqrt{E} \int_{r^{\prime}}^{r} \frac{d \bar{r}}{\left(1-E f(\bar{r})-\frac{J^{2} f(\bar{r})}{\bar{r}^{2}}\right)^{1 / 2}} \\
t-t^{\prime} & =-\int_{r^{\prime}}^{r} \frac{d \bar{r}}{f(\bar{r})\left(1-E f(\bar{r})-\frac{J^{2} f(\bar{r})}{\bar{r}^{2}}\right)^{1 / 2}} \\
\phi-\phi^{\prime} & =-J \int_{r^{\prime}}^{r} \frac{d \bar{r}}{\bar{r}^{2}\left(1-E f(\bar{r})-\frac{J^{2} f(\bar{r})}{\bar{r}^{2}}\right)^{1 / 2}}
\end{aligned}
$$


where $f(r) \equiv 1-2 M / r$. Note that $J=0$ yields a radial geodesic and $E=0$ a null one.

Expanding the integrand of Eq. (A7a in powers of $E$ and $J$, computing the integral, and then substituting into Eq. (A6) gives

$$
\sigma=-\frac{E}{2}\left(r^{\prime}-r\right)^{2}-\frac{E^{2}}{2} F\left(r^{\prime}-r\right)+O\left(E^{3}\right)+O\left(E J^{2}\right)
$$

with

$$
F \equiv-\int_{r^{\prime}}^{r} d \bar{r} f(\bar{r})=r^{\prime}-r-2 M \ln \left(\frac{r^{\prime}}{r}\right)
$$

The values of the constants $E$ and $J$ can be found by solving Eqs. (A7b) and (A7c). The integrals can be computed by first expanding the integrands in powers of $E$ and $J$. From Eq. (A7C) one finds

$$
J=\frac{r^{\prime} r}{r^{\prime}-r}\left(\phi-\phi^{\prime}\right)+O\left[E\left(\phi-\phi^{\prime}\right)\right]+O\left[\left(\phi-\phi^{\prime}\right)^{3}\right] .
$$

Keeping terms in the expansion of the integrand in Eq. (A7b to $O\left(J^{2}\right)$ and $O\left(E^{2}\right)$ and using (A10) yields

$$
\begin{aligned}
E= & -\frac{2\left(t^{\prime}-t_{R}\right)}{r^{\prime}-r}-\frac{r^{\prime} r}{\left(r^{\prime}-r\right)^{2}}\left(\phi-\phi^{\prime}\right)^{2}-\frac{3\left(t^{\prime}-t_{R}\right)^{2} F}{\left(r^{\prime}-r\right)^{3}} \\
& +O\left[\left(t^{\prime}-t_{R}\right)^{3}\right]+O\left[\left(t^{\prime}-t_{R}\right)\left(\phi-\phi^{\prime}\right)^{2}\right]+O\left[\left(\phi-\phi^{\prime}\right)^{4}\right]
\end{aligned}
$$

with

$$
t_{R}=t+\int_{r^{\prime}}^{r} \frac{d \bar{r}}{f(\bar{r})}=t-\left(r^{\prime}-r\right)-2 M \ln \frac{r^{\prime}-2 M}{r-2 M} .
$$

Substituting into Eq. (A8) gives

$$
\begin{aligned}
\sigma= & \left(t^{\prime}-t_{R}\right)\left(r^{\prime}-r\right)-\frac{F}{2\left(r^{\prime}-r\right)}\left(t^{\prime}-t_{R}\right)^{2}+\frac{r^{\prime} r}{2}\left(\phi-\phi^{\prime}\right)^{2} \\
& +O\left[\left(t^{\prime}-t_{R}\right)^{3}\right]+O\left[\left(t^{\prime}-t_{R}\right)\left(\phi-\phi^{\prime}\right)^{2}\right]+O\left[\left(\phi-\phi^{\prime}\right)^{4}\right] .
\end{aligned}
$$

Computing the various derivatives of $\sigma$ and then setting $t^{\prime}=t_{R}$ and $\phi^{\prime}=\phi$ gives

$$
\sigma_{t_{R}}=\left(r^{\prime}-r\right)
$$

and

$$
\begin{aligned}
\sigma_{; t t^{\prime}} & =\frac{F}{r^{\prime}-r} \\
\sigma_{; t r^{\prime}} & =-1+\frac{F}{f\left(r^{\prime}\right)\left(r^{\prime}-r\right)} \\
\sigma_{; r t^{\prime}} & =-1+\frac{F}{f(r)\left(r^{\prime}-r\right)} \\
\sigma_{; r r^{\prime}} & =-\frac{1}{f(r)}-\frac{1}{f\left(r^{\prime}\right)}+\frac{F}{f(r) f\left(r^{\prime}\right)\left(r^{\prime}-r\right)} \\
\sigma_{; \phi \phi^{\prime}} & =-r r^{\prime} .
\end{aligned}
$$


Using Eqs. (A4) and (A15) it is easy to show that

$$
\operatorname{det}\left[-\sigma_{; \mu \nu^{\prime}}\left(y, y_{R}\right)\right]=\left(r r_{0} \sin \theta_{0}\right)^{2}
$$

Substituting into Eq. (A1a) then gives

$$
u\left(y, y_{R}\right)=1
$$

This is an exact result.

\section{APPENDIX B: POWER SERIES EXPANSION FOR THE SELF-FORCE}

In this appendix an expansion for the self-force in powers of $r-r_{0}$ is derived. The coefficients of the expansion depend on the mass $M$ of the black hole, the radius $r_{0}$ at which the particle begins falling, the radius $r$ which is its present location, and the charge $q$.

To begin, consider $\Phi_{s}$ in either Eq. (19a) or (21). Its contribution to the self-force is given in Eq. (201). From Eq. (A12) it is clear that $\partial_{t}=\partial_{t_{R}}$. Because $v\left(x, x_{1}\right)$ satisfies the equation $\square_{x} v=0$ and because of the time translation and time reversal invariance of the metric (8), $v$ is a function of $\left(t-t_{1}\right)^{2}$. Making use of this fact one finds that

$$
\begin{aligned}
\frac{\partial \Phi_{s}}{\partial t} & =\frac{q}{8 \pi} \sqrt{1-\frac{2 M}{r_{0}}}\left[v\left(t, r ; t_{R}, r_{0}\right)-\int_{0}^{t_{R}} \frac{\partial}{\partial t_{1}} v\left(t, r ; t_{1}, r_{0}\right) d t_{1}\right] \\
& =\frac{q}{8 \pi} \sqrt{1-\frac{2 M}{r_{0}}} v\left(t, r ; 0, r_{0}\right) .
\end{aligned}
$$

Here as in Section \II we suppress the dependence of $v$ on $\theta_{0}$ and $\phi_{0}$.

Next consider $\Phi_{f}$ in Eq. (19b). The geodesic equations (A7) can be used to change the integration variable from the proper time to the coordinate time. In this case $J=0$ as the geodesic is radial. Since it starts from rest at $r_{0}$ it can be seen from the geodesic equations that $E=\left(1-2 M / r_{0}\right)^{-1}$. The result is

$$
\Phi_{f}=\frac{q}{8 \pi} \frac{1}{\sqrt{1-\frac{2 M}{r_{0}}}} \int_{0}^{t} v\left(t, r ; t_{1}, r_{1}\right)\left(1-\frac{2 M}{r_{1}}\right) \mathrm{dt}_{1} .
$$

Noting that in a Schwarzschild spacetime $v(x, x)=0$, one finds

$$
\frac{\partial \Phi_{f}}{\partial t}=-\frac{q}{8 \pi} \frac{1}{\sqrt{1-\frac{2 M}{r_{0}}}} \int_{0}^{t}\left(\frac{\partial v\left(t, r ; t_{1}, r_{1}\right)}{\partial t_{1}}\right)\left(1-\frac{2 M}{r_{1}}\right) d t_{1}
$$


Since the particle is freely falling, $r_{1}=r_{1}\left(t_{1}\right)$ and one can write

$$
\frac{\partial v}{\partial t_{1}}\left(1-\frac{2 M}{r_{1}}\right)=\frac{d}{d t_{1}}\left[v\left(1-\frac{2 M}{r_{1}}\right)\right]-\frac{d r_{1}}{d t_{1}} \frac{\partial}{\partial r_{1}}\left[v\left(1-\frac{2 M}{r_{1}}\right)\right]
$$

with the result that

$$
\begin{aligned}
\frac{\partial \Phi_{f}}{\partial t}=\frac{q}{8 \pi} & \frac{1}{\sqrt{1-\frac{2 M}{r_{0}}}}\left\{v\left[t, r ; 0, r_{0}\right]\left(1-\frac{2 M}{r_{0}}\right)\right. \\
& \left.+\int_{r_{0}}^{r} \frac{\partial}{\partial r_{1}}\left[v\left(t, r ; t_{1}, r_{1}\right)\left(1-\frac{2 M}{r_{1}}\right)\right] d r_{1}\right\} .
\end{aligned}
$$

Thus

$$
f_{t}=-\frac{q^{2}}{8 \pi} \frac{1}{\sqrt{1-\frac{2 M}{r_{0}}}} \int_{r_{0}}^{r} \frac{\partial}{\partial r_{1}}\left[v\left(t, r ; t_{1}, r_{1}\right)\left(1-\frac{2 M}{r_{1}}\right)\right] d r_{1}
$$

The computation of $f_{r *}$ is straightforward. Taking the derivative of Eqs. (21) and (19b) and then using the geodesic Eqs. (A7) and (23) one finds

$$
\begin{aligned}
f_{r *}= & \frac{q^{2}}{8 \pi} \sqrt{1-\frac{2 M}{r_{0}}}\left(1-\frac{2 M}{r}\right) \sum_{i, k=0}^{\infty}\left\{\frac{1}{2 i+1}\right. \\
& \left.\times\left[\frac{d v_{i 0 k}}{d r}\left(r-r_{0}\right)^{k}+k v_{i 0 k}\left(r-r_{0}\right)^{k-1}\right]\left[t^{2 i+1}-\left(t-t_{R}\right)^{2 i+1}\right]\right\} \\
& +\frac{q^{2}}{8 \pi}\left(1-\frac{2 M}{r}\right) \sqrt{\frac{r_{0}}{2 M}} \int_{r_{0}}^{r} \frac{\partial v\left(t, r ; t_{1}, r_{1}\right)}{\partial r} \sqrt{\frac{r_{1}}{r_{0}-r_{1}}} d r_{1} .
\end{aligned}
$$

The next step is to expand $t$ in powers of $r_{0}-r$ and $t_{1}$ in powers of $r_{0}-r_{1}$. This is done using the geodesic equation $(\underline{\mathrm{A}} \mathrm{b} \mathrm{b})$. Then making the change of variables

$$
\begin{aligned}
s & =\sqrt{\frac{r_{0}-r}{r_{0}}} \\
s_{1} & =\sqrt{\frac{r_{0}-r_{1}}{r_{0}}} \\
x_{0} & =\sqrt{\frac{2 M}{r_{0}}} \\
w_{0} & =\sqrt{1-\frac{2 M}{r_{0}}}
\end{aligned}
$$

gives

$$
t=\frac{r_{0}}{x_{0}}\left[\frac{2 s}{w_{0}}+\left(\frac{2}{3}-w_{0}^{2}\right)\left(\frac{s}{w_{0}}\right)^{3}+\ldots\right]
$$


Substituting this and the corresponding expression for $t_{1}$ into Eqs. (B6) and (B7), expanding in powers of $s$ and $s_{1}$, and computing the integrals gives

$$
\begin{aligned}
f_{t} & =q^{2} \sum_{n=3}^{\infty} a_{2 n} s^{2 n} \\
f_{r *} & =q^{2} \sum_{n=2}^{\infty} b_{2 n+1} s^{2 n+1} .
\end{aligned}
$$

Using a $16^{\text {th }}$ order WKB expansion for $v\left(x, x^{\prime}\right)$ we find

$$
\begin{aligned}
a_{6}= & \frac{w_{0}\left(-13+53 w_{0}^{2}\right)}{22400 \pi r_{0}^{2}} \\
a_{8}= & \frac{w_{0}}{470400 \pi r_{0}^{2} x_{0}^{2}}\left(-703+2031 w_{0}^{2}+72 w_{0}^{4}\right) \\
a_{10}= & \frac{w_{0}}{155232000 \pi r_{0}^{2} x_{0}{ }^{4}}\left(-404385+1485889 w_{0}^{2}-1990813 w_{0}^{4}+1497309 w_{0}^{6}\right) \\
a_{12}= & \frac{w_{0}}{355170816000 \pi r_{0}^{2} x_{0}{ }^{6}}\left(-1380534784+6209685607 w_{0}^{2}-9489117981 w_{0}^{4}\right. \\
& \left.+2956643877 w_{0}^{6}+3255643281 w_{0}^{8}\right) \\
a_{14}= & \frac{w_{0}}{129282177024000 \pi r_{0}^{2} x_{0}^{8}}\left(-690105799163+3725808468235 w_{0}^{2}\right. \\
& -7969067122446 w_{0}^{4}+9871663249718 w_{0}^{6}-9633792321223 w_{0}^{8} \\
& \left.+5317042452879 w_{0}{ }^{10}\right) \\
a_{16}= & -\frac{w_{0}}{131867820564480000 \pi r_{0}^{2} x_{0}^{10}}\left(917523531344444-5807610696686037 w_{0}^{2}\right. \\
& +15120303760167717 w_{0}^{4}-19548426682161946 w_{0}^{6}+7163353443413246 w_{0}^{8} \\
& \left.+11432898205974399 w_{0}{ }^{10}-9956772991427823 w_{0}^{12}\right)
\end{aligned}
$$


and

$$
\begin{aligned}
b_{5}= & -\frac{3 x_{0} w_{0}^{2}}{11200 \pi r_{0}^{2}} \\
b_{7}= & \frac{1}{470400 \pi x_{0} r_{0}^{2}}\left(-147+1031 w_{0}^{2}-2004 w_{0}^{4}\right) \\
b_{9}= & \frac{1}{558835200 \pi x_{0}^{3} r_{0}^{2}}\left(725274-4840427 w_{0}^{2}+7544064 w_{0}^{4}-1384911 w_{0}^{6}\right) \\
b_{11}= & \frac{1}{1598268672000 \pi x_{0}^{5} r_{0}^{2}}\left(-5192394350+35645887586 w_{0}^{2}-76724155827 w_{0}^{4}\right. \\
& \left.+74055537336 w_{0}^{6}-34933610745 w_{0}^{8}\right) \\
b_{13}= & \frac{1}{13851661824000 \pi x_{0}^{7} r_{0}^{2}}\left(88650418610-652839443586 w_{0}^{2}+1715470441205 w_{0}^{4}\right. \\
& \left.-2048237904519 w_{0}^{6}+938994638717 w_{0}^{8}+27641613573 w_{0}{ }^{10}\right) \\
b_{15}= & \frac{1}{98900865423360000 \pi x_{0}^{9} r_{0}^{2}}\left(-1082712168000450+8690022729803252 w_{0}^{2}\right. \\
& -27039642594514215 w_{0}^{4}+43159798466443548 w_{0}^{6}-40035939562445204 w_{0}^{8} \\
& \left.+24271024582691064 w_{0}^{10}-8499901220531595 w_{0}^{12}\right) .
\end{aligned}
$$

[1] B. S. DeWitt and R. W. Brehme, Ann. Phys. (N.Y.) 9, 220 (1960); J. M. Hobbs, Ann. Phys. (NY) 47, 141 (1968); Y. Mino, M. Sasaki, and T. Tanaka, Phys. Rev. D 55, 3457 (1997); T. C. Quinn and R. M. Wald, Phys. Rev. D 56, 3381 (1997).

[2] T. C. Quinn, Phys. Rev. D 62, 064029 (2000).

[3] For excellent reviews, see E. Poission, gr-qc/0306052; S. Detweiler, Classical Quantum Gravity 22, S681 (2005).

[4] B. F. Schutz. Gravitational wave astronomy. Class. Quant. Grav. 16 A131 (1999).

[5] S. A. Hughes. Listening to the universe with gravitational-wave astronomy. Annals. Phys. 303, 142 (2003).

[6] A. G. Smith and C. M. Will, Phys. Rev. D 22, 1276 (1980).

[7] D. Lohiya, J. Phys. A: Math. Gen. ,15, 1815 (1982)

[8] A. G. Wiseman, Phys. Rev. D 61, 084014 (2000).

[9] E. Rosenthal, Phys. Rev. D 69064035 (2004); D70, 124016(2004)

[10] B. Leaute and B. Linet, J. Phys. A 15, 1821 (1982).

[11] F. Piazzese and G. RIZZI, Gen. Rel. and Grav. 23, 403 (1991). 
[12] C. M. DeWitt and B. S. DeWitt, Physics 1, 3 (1964).

[13] D. V. Gal'tsov, J. Phys. A 15, 3737 (1982).

[14] M. J. Pfenning and E. Poisson, Phys. Rev. D 65084001 (2002).

[15] L. Barack and A. Ori, Phys. Rev. D 61, 061502(R) (2000).

[16] L. Barack, Y. Mino, H. Nakano, A. Ori, and M. Sasaki, Phys. Rev. Lett. 88091101 (2002).

[17] L. M. Burko, Class. Quant. Grav. 17, 227 (2000).

[18] L. M. Burko, Y. T. Liu, and Y. Soen, Phys. Rev. D 63, 024015 (2001).

[19] L. M. Burko and Y. T. Liu, Phys. Rev. D64, 024006 (2001).

[20] L. Barack and L. M. Burko, Phys. Rev. D 62, 084040 (2000).

[21] L. Barack and C. O. Lousto, Phys. Rev. D 66 061502(R) (2002).

[22] L. M. Burko, Phys. Rev. Lett. 84, 4529 (2000).

[23] S. Detweiler, E. Messaritaki, and B. F. Whiting, Phys. Rev. D 67104016 (2003).

[24] L. M. Diaz-Rivera, E. Messaritaki, B. F. Whiting, and S. Detweiler, Phys. Rev. D 70, 124018 (2004).

[25] W. Hikida, S. Jhingan, H. Nakano, N. Sago, M. Sasaki, T. Tanaka, Prog. Theor. Phys. 113, $283(2005)$.

[26] C. O. Lousto, Phys. Rev. Lett. 84, 5251 (2000).

[27] S. Detweiler and E. Poisson, Phys. Rev. D 69, 084019 (2004).

[28] P. R. Anderson and B. L. Hu, Phys. Rev. D 69, 064039 (2004). Paper I.

[29] J. Schwinger, Phys. Rev. 82, 664 (1951).

[30] B. S. DeWitt, in Relativity, Groups and Topology, edited by B. S. DeWitt and C. DeWitt (Gordon and Breach, New York, 1965); Phys. Rep. 19, 295 (1975).

[31] S. M. Christensen, Phys. Rev. D 14, 2490 (1976).

[32] S. L. Adler, J. Lieberman and Y. J. Ng, Ann. Phys. (N.Y.) 106, 279 (1977).

[33] R. M. Wald, Commun. Math. Phys. 45, 9 (1975); Phys. Rev. D17, 1477 (1978).

[34] P. R. Anderson, W. A. Hiscock, and D. A. Samuel, Phys. Rev. Lett. 70, 1739 (1993); Phys. Rev. D 51, 4337 (1995).

[35] N. G. Phillips and B. L. Hu, Phys. Rev. D63 104001 (2001); D67 104002 (2003).

[36] W. G. Anderson, E. E. Flanagan, A. C. Ottewill, Phys.Rev. D 71024036 (2005).

[37] W. G. Anderson and A. G. Wiseman, Classical Quantum Gravity 22, S783 (2005).

[38] To see this, note that $\cos n \gamma=\frac{1}{2} \sum_{k=0}(-1)^{k} \frac{(n-k) !}{k !(n-2 k) !}(2 \cos \gamma)^{n-2 k} ;$ M. R. Spiegel, Mathemati- 
cal handbook of formulas and tables, (McGraw-Hill, New York, 1968), pp. 17.

[39] S. Weinberg, Gravitation and Cosmology: Principles and Applications of the General Theory of Relativity, (John Wiley 8 Sons, Inc., 1972), pp. 185-188.

[40] P. R. Anderson, A. Eftekharzadeh, and B. L. Hu, Phys. Rev. D73 064023 (2006).

[41] P. R. Anderson, A. Eftekharzadeh, and B. L. Hu, Phys. Rev. D77, 089902(E) (2008).

[42] P. R. Anderson and B. L. Hu, Phys. Rev. D 77, 089901(E) (2008).

\section{APPENDIX C: ERRATUM}

In the Physical Review D (PRD) version of this paper [40] there were errors due to a factor of 2 error in Paper I [28]. These errors have been corrected in this arXiv version and also published in an erratum [41]. For completeness we list the corrections in that erratum here as well.

In Ref. [28] there was a factor of two error in the calculation of the coefficients of the expansion for $v\left(x, x^{\prime}\right)$ that has been corrected in Ref. [42]. This expansion, carried to higher order, was used in the calculations of Ref. [40]. We correct the resulting errors in Ref. [40] here.

Eqs. (22.a) and (22.b) should be multiplied by a factor of 2 with the result

$$
\begin{aligned}
f_{t}(\tau) & =\frac{q^{2}}{22400 \pi r_{0}^{2}} \sqrt{1-\frac{2 M}{r_{0}}}\left(40-\frac{106 M}{r_{0}}\right)\left(\frac{r_{0}-r}{r_{0}}\right)^{3}+O\left[\left(\frac{r_{0}-r}{r_{0}}\right)^{4}\right] \\
f_{r *}(\tau) & =-\frac{3 q^{2}}{11200 \pi r_{0}^{2}} \sqrt{\frac{2 M}{r_{0}}}\left(1-\frac{2 M}{r_{0}}\right)\left(\frac{r_{0}-r}{r_{0}}\right)^{5 / 2}+O\left[\left(\frac{r_{0}-r}{r_{0}}\right)^{7 / 2}\right]
\end{aligned}
$$

The entries in columns 2 and 4 of Table 1 should be multiplied by a factor of 2 . The revised results are shown in Table 1. The curves in Figure 1 need to be multiplied by a factor of 2. The revised figure is shown in Figure 1. 


\begin{tabular}{|c|c|c|c|c|}
\hline$r / M$ & $10^{14}\left(M^{2} / q^{2}\right) f_{t}$ & Error & $10^{14}\left(M^{2} / q^{2}\right) f_{r^{*}}$ & Error \\
\hline 34.25 & 6.93066 & $6.9 \times 10^{-6}$ & -5.7384 & $3.4 \times 10^{-5}$ \\
\hline 34.00 & 120.72 & $4.4 \times 10^{-4}$ & -96.78 & $1.4 \times 10^{-3}$ \\
\hline 33.75 & 616 & $3.4 \times 10^{-3}$ & -520 & $8.4 \times 10^{-3}$ \\
\hline 33.50 & 2080 & 0.013 & -1840 & 0.25 \\
\hline 33.25 & 5680 & 0.031 & -5200 & 0.052 \\
\hline 33.00 & 13800 & 0.059 & -13200 & 0.089 \\
\hline 32.75 & 30000 & 0.10 & -30000 & 0.13 \\
\hline 32.50 & 66000 & 0.14 & -62000 & 0.18 \\
\hline
\end{tabular}

TABLE II: The dependence of the temporal $\left(f_{t}\right)$ and radial $\left(f_{r^{*}}\right)$ components of the self-force on the radial distance $r / M$ are given for a particle undergoing radial infall after being held at rest until a time $t=0$. The particle's initial location is at $r^{*}=40 M$ which corresponds to $r \approx 34.43 M$. The error shown for each case is an estimate of the relative error and is obtained by taking the absolute value of the ratio of the last term used in the series for the self-force to the entire series.

Equations (B10c) and (B10d) need to be multiplied by a factor of 2 . The result is

$$
\begin{aligned}
& a_{6}=\frac{w_{0}\left(-13+53 w_{0}^{2}\right)}{22400 \pi r_{0}^{2}} \\
& a_{8}=\frac{w_{0}}{470400 \pi r_{0}^{2} x_{0}^{2}}\left(-703+2031 w_{0}^{2}+72 w_{0}^{4}\right) \\
& a_{10}=\frac{w_{0}}{155232000 \pi r_{0}^{2} x_{0}{ }^{4}}\left(-404385+1485889 w_{0}^{2}-1990813 w_{0}^{4}+1497309 w_{0}^{6}\right) \\
& a_{12}=\frac{w_{0}}{355170816000 \pi r_{0}{ }^{2} x_{0}{ }^{6}}\left(-1380534784+6209685607 w_{0}^{2}-9489117981 w_{0}^{4}\right. \\
& \left.+2956643877 w_{0}^{6}+3255643281 w_{0}^{8}\right) \\
& a_{14}=\frac{w_{0}}{129282177024000 \pi r_{0}^{2} x_{0}^{8}}\left(-690105799163+3725808468235 w_{0}^{2}\right. \\
& -7969067122446 w_{0}^{4}+9871663249718 w_{0}{ }^{6}-9633792321223 w_{0}^{8} \\
& \left.+5317042452879 w_{0}^{10}\right) \\
& a_{16}=-\frac{w_{0}}{131867820564480000 \pi r_{0}^{2} x_{0}^{10}}\left(917523531344444-5807610696686037 w_{0}^{2}\right. \\
& +15120303760167717 w_{0}^{4}-19548426682161946 w_{0}^{6}+7163353443413246 w_{0}^{8} \\
& \left.+11432898205974399 w_{0}^{10}-9956772991427823 w_{0}^{12}\right)
\end{aligned}
$$




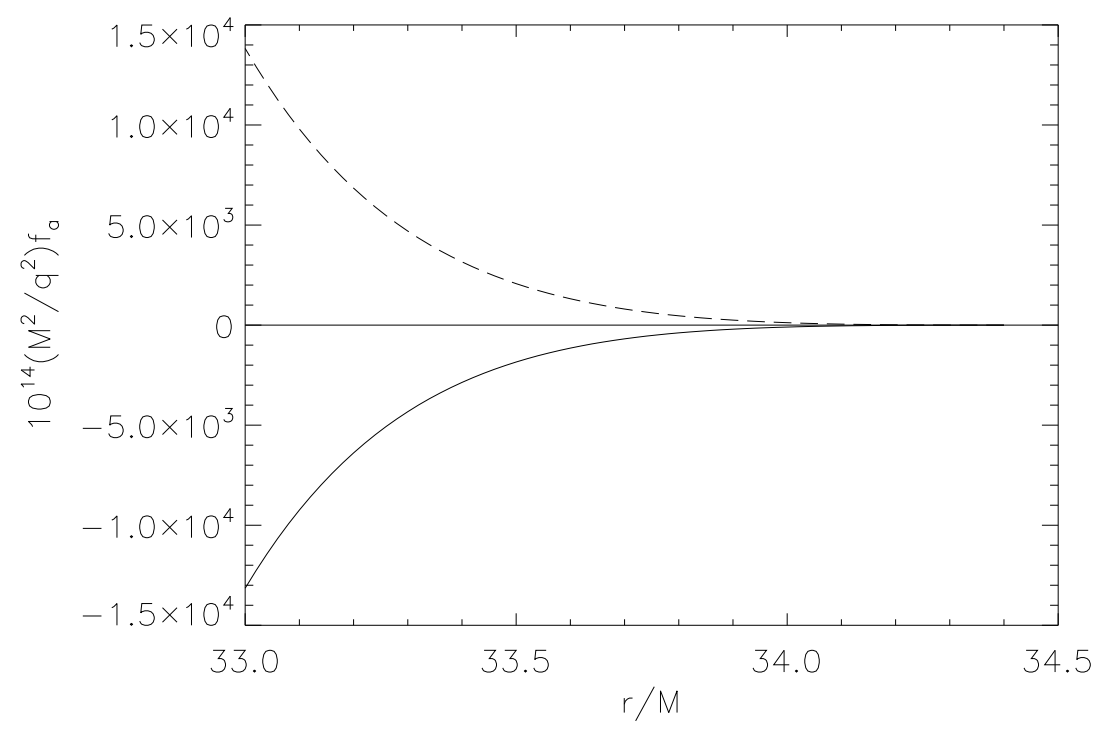

FIG. 2: In this figure the two components of the self-force are plotted for the case of a particle undergoing radial infall after being held at rest until a time $t=0$. The particle's initial location is at $r^{*}=40 M$ which corresponds to $r \approx 34.43 M$. The dashed curve corresponds to $f_{t}$ and the solid curve to $f_{r^{*}}$.

and

$$
\begin{aligned}
b_{5}= & -\frac{3 x_{0} w_{0}^{2}}{11200 \pi r_{0}^{2}} \\
b_{7}= & \frac{1}{470400 \pi x_{0} r_{0}^{2}}\left(-147+1031 w_{0}^{2}-2004 w_{0}^{4}\right) \\
b_{9}= & \frac{1}{558835200 \pi x_{0}^{3} r_{0}^{2}}\left(725274-4840427 w_{0}^{2}+7544064 w_{0}^{4}-1384911 w_{0}^{6}\right) \\
b_{11}= & \frac{1}{1598268672000 \pi x_{0}^{5} r_{0}^{2}}\left(-5192394350+35645887586 w_{0}^{2}-76724155827 w_{0}^{4}\right. \\
& \left.+74055537336 w_{0}^{6}-34933610745 w_{0}^{8}\right) \\
b_{13}= & \frac{1}{13851661824000 \pi x_{0}^{7} r_{0}^{2}}\left(88650418610-652839443586 w_{0}^{2}+1715470441205 w_{0}^{4}\right. \\
& \left.-2048237904519 w_{0}^{6}+938994638717 w_{0}^{8}+27641613573 w_{0}{ }^{10}\right) \\
b_{15}= & \frac{1}{98900865423360000 \pi x_{0}^{9} r_{0}^{2}}\left(-1082712168000450+8690022729803252 w_{0}^{2}\right. \\
& -27039642594514215 w_{0}^{4}+43159798466443548 w_{0}^{6}-40035939562445204 w_{0}^{8} \\
& \left.+24271024582691064 w_{0}^{10}-8499901220531595 w_{0}^{12}\right) .
\end{aligned}
$$


We would like to thank Barry Wardell for alerting us to the possibility that a factor of two error existed in our results in Ref. [42] and for help in finding it. This work was supported in part by the National Science Foundation under grant numbers PHY03-00710 and PHY05-56292. 